\title{
Guidelines
}

\section{UK Guidelines for the Management of Bone Sarcomas}

\author{
Robert Grimer, ${ }^{1}$ Nick Athanasou, ${ }^{2}$ Craig Gerrand, ${ }^{3}$ Ian Judson, ${ }^{4}$ Ian Lewis, ${ }^{5}$ Bruce Morland, ${ }^{6}$ \\ David Peake, ${ }^{7}$ Beatrice Seddon, ${ }^{8}$ and Jeremy Whelan ${ }^{8}$
}

${ }^{1}$ Royal Orthopaedic Hospital, Birmingham B31 2AP, UK

${ }^{2}$ Nuffield Orthopaedic Centre, Oxford OX3 7LD, UK

${ }^{3}$ Freeman Hospital, Newcastle-upon-Tyne NE7 7DN, UK

${ }^{4}$ The Royal Marsden, Sutton SM2 5PT, UK

${ }^{5}$ Leeds Teaching Hospitals, LS9 7TF, UK

${ }^{6}$ Birmingham Children's Hospital, B4 6NH, UK

${ }^{7}$ Queen Elizabeth Hospital, Birmingham B15 2TH, UK

${ }^{8}$ University College Hospital, London NW1 2BU, UK

Correspondence should be addressed to Robert Grimer, rob.grimer@btopenworld.com

Received 10 September 2010; Accepted 20 October 2010

Academic Editor: Ole Nielsen

Copyright (C) 2010 Robert Grimer et al. This is an open access article distributed under the Creative Commons Attribution License, which permits unrestricted use, distribution, and reproduction in any medium, provided the original work is properly cited.

\begin{abstract}
These guidelines have been developed in order to provide an overview and a set of broad-based key recommendations for the management of patients with bone sarcomas in the UK. They have taken into consideration the most up-to-date scientific literature along with the recent recommendations by the European Society of Medical Oncology. The principles of the NICE guidance on both "improving outcomes for patients with sarcomas" and "improving outcomes with children and young people with cancer" have been incorporated. As care evolves, it is acknowledged that these guidelines will need updating. The key recommendations are that bone pain or a palpable mass should always lead to further investigation and patients with clinicoradiological findings suggestive of a primary bone tumour should be sent to a reference centre. Patients should then have their care managed at such a specialist centre by a fully accredited multidisciplinary team.
\end{abstract}

\section{Introduction}

1.1. Rationale and Objective of Guidelines. Bone sarcomas are an uncommon group of malignancies. It was recognised more than 20 years ago in England that management of bone sarcomas should be centralised; this led to the recognition of two supraregional centres by the National Specialist Commissioning Advisory Group (NSCAG) [now National Commissioning Group (NCG)] in 1986. Until now, however, there have been no clinical guidelines to document the standard of care for patients with these tumours.

In the US, the National Comprehensive Cancer Network (NCCN) bone sarcoma guidelines [1] are highly regarded as are those developed by The European Society of Medical Oncology (ESMO) in conjunction with EUROBONET [24]. Using these documents as a framework, clinical management guidelines for patients with bone sarcoma in the UK were drawn up by consensus under the auspices of the British Sarcoma Group (BSG). Levels of evidence $[\mathrm{I}-\mathrm{V}]$ and grades of recommendation $[\mathrm{A}-\mathrm{D}]$ as used by the American Society of Clinical Oncology [5] have been published recently in the ESMO guidelines and are also referred to elsewhere $[6,7]$. We have not included the levels of evidence in this paper.

These guidelines are not intended to be prescriptive, but aim to educate and improve the quality of care for patients with bone sarcomas by helping to identify and inform the key decisions involved in their management. Equally, this paper does not extend to rehabilitation, prosthetic services, and palliation.

1.2. Methods. The NCCN and ESMO guidelines together with the National Institute for Health and Clinical Excellence (NICE) Improving Outcomes Guidance for people with sarcoma [8] were used as the basis for discussion by the group, adding or omitting detail only where it was clearly agreed by consensus, in relation to UK specific issues. A recent literature review has been included to ensure that referencing is comprehensive. 
1.3. Scope of Guidelines. The recommendations apply to all bone sarcomas arising in any skeletal location.

These guidelines focus on clinical effectiveness, giving a picture of what treatments a specialist sarcoma multidisciplinary team (MDT) should have access to within the UK, subject to some flexibility to allow for evolving practice, but they do not employ the same detailed analysis of cost effectiveness as NICE. This material can be considered to represent a broad consensus in 2010; however, we acknowledge that it will require updating as treatment evolves.

\section{Overview}

2.1. Classification and Clinical Presentation. Primary bone tumours are rare, accounting for less than $1 \%$ of cancers in adults [9]. There are on average 427 new cases per year in England and Wales [8] Data from the Office of National Statistics and Welsh Cancer Intelligence and Surveillance Unit. They have a relatively high incidence in children and adolescents (accounting for approximately $5 \%$ of all childhood cancers in European Countries) [10], but can arise at any age. Given their rarity, nonspecialised clinicians, radiologists, and pathologists may find them difficult to recognise. To avoid diagnostic and management difficulties early referral to a specialist MDT prior to biopsy is important [11].

In adulthood, metastatic carcinomas and haemopoietic malignancies in bone considerably outnumber primary bone tumours. Where there is diagnostic uncertainty, it should be assumed that the patient has a primary bone sarcoma until proven otherwise [12].

During a working lifetime, a General Practitioner (GP) is unlikely to see a patient with a bone sarcoma. Delays in diagnosis are, therefore, common and earlier diagnosis would almost certainly lead to improved outcomes both in terms of survival and less extensive surgery.

The most common symptom of a primary bone tumour is pain which may be nonmechanical or night pain. The presence of bone pain at night should always be considered to be a "red flag" symptom leading to further investigation. The presence of bone swelling or a soft-tissue mass may occur later. The average duration of symptoms is 3 months although a history of 6 months or longer is not uncommon [13-15]. The presence of pain or a palpable mass arising from any bone should cause concern and lead to further investigation of which a plain X-ray is the first investigation of choice. The presence of any of the following on the X-ray is suggestive, but not diagnostic of a bone tumour and should also lead to further investigation:

(i) bone destruction,

(ii) new bone formation,

(iii) periosteal swelling,

(vi) soft tissue swelling.

The histological classification of primary malignant bone tumours according to the World Health Organisation (WHO) is given in Table 1.
Although listed by the WHO as bone tumours, the management of haemopoetic tumours of bone is beyond the scope of these guidelines.

2.2. Epidemiology and Aetiology. Although there are a number of inherited and acquired factors associated with the development of primary bone tumours, it is not possible to identify a particular cause in the majority of patients $[17,18]$.

2.2.1. Osteosarcoma. Osteosarcoma is the most frequent primary cancer of bone (incidence $0.2-0.3 / 100,000 /$ year) [2], UK rates are 0.27/100,000 population in England [19]. There is an average of between 124-150 cases per year in England and Wales $[8,19]$. The incidence is higher in adolescents $(0.8-1.1 / 100,000 /$ year at age 15-19), where it accounts for $>10 \%$ of all solid cancers. The male-female ratio is $1.4: 1$ (UK data 1.1:1-NCIN) The majority arise in adolescence, but some are linked to other pathologies in the seventh and eighth decades of life [20].

Osteosarcoma usually arises in the metaphysis of an extremity long bone, most commonly around the knee [21, 22]. Some tumours (predominantly in adults) arise in the axial skeleton or craniofacial bones. Conventional osteosarcoma, a high-grade malignancy, accounts for the majority of osteosarcoma. Its most frequent subtypes are osteoblastic, chondroblastic, and fibroblastic. Other high-grade types are telangiectatic, small cell, and high-grade surface osteosarcoma. Low-grade central and parosteal osteosarcoma are low-grade malignancies, while periosteal osteosarcoma is an intermediate-grade chondroblastic osteosarcoma. Risk factors for the occurrence of osteosarcoma include previous radiation therapy, Paget's disease of bone, and germ line abnormalities such as the Li-Fraumeni syndrome, Werner syndrome, Rothmund-Thomson syndrome, and familial retinoblastoma $[23,24]$.

2.2.2. Ewing Sarcoma. Ewing sarcoma (including primitive neuroectodermal tumour of bone) is the second most common primary malignant bone cancer in children and adolescents, but is also seen in adults. The median age at diagnosis is also around 15 years and there is a male predilection of $1.5: 1$ (UK data 1.2:1 NCIN). Ewing sarcoma is diagnosed in children aged $0-20$ at an incidence of $0.3 / 100,000 /$ year [UK all age standardised rates report $0.11-0.12 / 100,000$ population in the UK (1979-2004)] with approximately 65-75 new cases per year in the UK $[8,19]$. The most frequent sites of involvement are the long bones, pelvis, ribs, and vertebral column. It is less common in people of Chinese or Black African origin. All forms of Ewing sarcoma are high-grade tumours $[3,25]$.

2.2.3. Chondrosarcoma. Chondrosarcoma is one of the most common bone sarcomas of adulthood, characterised by the production of tumour cartilage [26]. The age standardised incidence may be as high as $0.25 / 100,000$ per year in males and $0.2 / 100,000$ in females per year [UK age standardised rates report $0.19 / 100,000$ population in the UK (NCIN)], with the most common age being between 30-60 years [27]. 
TABLE 1: 2002 WHO classification of malignant bone tumours [16].

\begin{tabular}{|c|c|c|}
\hline \multirow{12}{*}{ Osteogenic tumours } & Osteosarcoma & $9180 / 3$ \\
\hline & Conventional & $9180 / 3$ \\
\hline & Chondroblastic & $9181 / 3$ \\
\hline & Fibroblastic & $9182 / 3$ \\
\hline & Osteoblastic & $9180 / 3$ \\
\hline & Telangiectatic & $9183 / 3$ \\
\hline & Small cell & $9185 / 3$ \\
\hline & Low-grade central & $9187 / 3$ \\
\hline & Secondary & $9180 / 3$ \\
\hline & Parosteal & $9192 / 3$ \\
\hline & Periosteal & $9193 / 3$ \\
\hline & High grade surface & $9194 / 3$ \\
\hline Ewing sarcoma/primitive neuroectodermal tumour & Ewing sarcoma & $9260 / 3$ \\
\hline \multirow{6}{*}{ Cartilage } & Chondrosarcoma & $9220 / 3$ \\
\hline & Central, primary, and secondary & $9220 / 3$ \\
\hline & Peripheral & $9221 / 3$ \\
\hline & Differentiated & $9243 / 3$ \\
\hline & Mesenchymal & $9240 / 3$ \\
\hline & Clear cell & $9242 / 3$ \\
\hline Fibrogenic tumours & Fibrosarcoma & $8810 / 3$ \\
\hline \multicolumn{3}{|l|}{ Fibrohistiocytic tumours } \\
\hline \multirow{2}{*}{ Haemopoietic tumours } & Plasma cell myeloma & $9732 / 3$ \\
\hline & Malignant lymphoma, NOS & $9590 / 3$ \\
\hline Giant cell tumour & Malignancy in giant cell tumour & $9250 / 3$ \\
\hline Notochordal tumours & Chordoma & $9370 / 3$ \\
\hline Vascular tumours & Angiosarcoma & $9120 / 3$ \\
\hline Smooth muscle tumours & Leiomyosarcoma & $8890 / 3$ \\
\hline Lipogenic tumours & Liposarcoma & $8850 / 3$ \\
\hline Miscellaneous tumours & Adamantinoma & $9261 / 3$ \\
\hline
\end{tabular}

There are approximately $100-120$ new cases per year in the UK [8].

The majority of primary chondrosarcomas are low rather than high grade [28]. Most chondrosarcomas are located in the long bones, but they also occur in flat bones such as pelvis, rib, and scapula. Chondrosarcoma can arise in preexisting benign lesions such as osteochondroma and enchondroma. In these circumstances, they are referred to as secondary peripheral chondrosarcomas and secondary chondrosarcomas, respectively.

The majority of chondrosarcomas are of the conventional subtype, but rarer subtypes include mesenchymal chondrosarcoma and clear cell chondrosarcoma. In rare circumstances, conventional chondrosarcomas can "dedifferentiate" into a very high-grade tumour with a dismal prognosis socalled dedifferentiated chondrosarcoma [26, 29, 30] Most chondrosarcomas are solitary, but they can occur as multiple lesions in patients with osteochondromatosis (hereditary multiple exostoses) and enchondromatosis (Ollier's disease). The risk of malignancy in solitary enchondromas and osteochondromas is uncertain, but it is increased when there are multiple lesions.
2.2.4. Spindle Cell Sarcomas of Bone. Spindle cell sarcomas of bone represent between $2 \%$ and $5 \%$ of primary bone malignancies and include a variety of diagnostic groups including fibrosarcoma, malignant fibrous histiocytoma $(\mathrm{MFH})$, leiomyosarcoma, and undifferentiated sarcoma. These tumours arise in a similar age group to chondrosarcoma, but the skeletal distribution is more like that of osteosarcoma. They typically present with pain and have a high incidence of fracture at presentation. Radiological characteristics include a punched out or lytic appearance. An association with pre-existing disease/abnormality (Paget's disease or bone infarct) or history of previous irradiation has been reported. It is not unusual for a spindle cell sarcoma to be found to be either a dedifferentiated chondrosarcoma or an osteosarcoma after resection.

2.2.5. Other Bone Sarcomas. These include such entities as adamantinoma and chordoma which both have specific clinical presentation and management [16]. Adamantinoma of bone typically arises in the anterior cortex of the diaphysis of the tibia. Chordomas are rare, typically arising in the sacrum or base of skull from notochord remnants. 


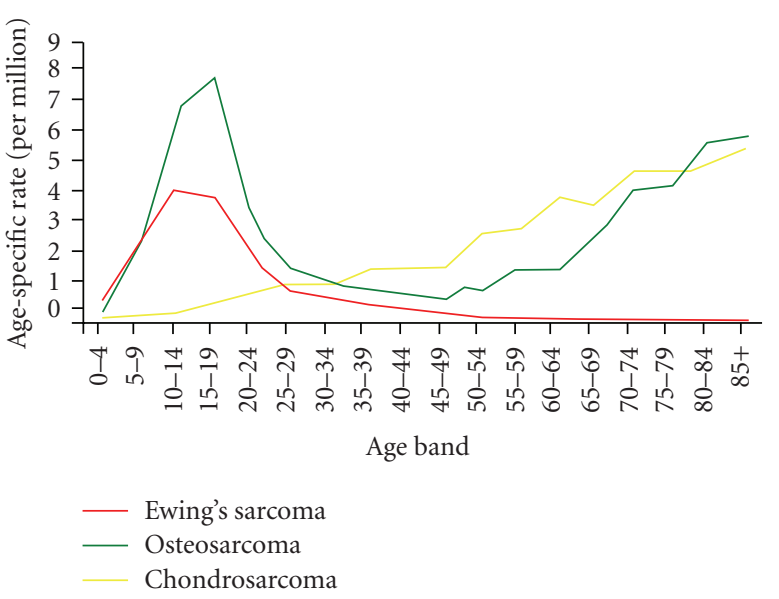

Figure 1: Age-specific incidence rates by morphology, England, 1979-2004.

Age-specific incidence rates by morphology are shown in Figure 1.

\section{Diagnosis and Referral}

3.1. Early Steps in Diagnosis. In children under the age of 5 years, primary malignant bone tumours are rare. Between the age of 5 and approximately 40, a primary malignant bone tumour is likely to be the most common diagnosis of a bone neoplasm in the absence of a past history of malignancy. Over 40, the incidence of metastases (usually from bronchus, breast, thyroid, kidney, or prostate) becomes increasingly common. At any age, the possibility of a benign lesion, infection, or haematological malignancy (plasma cell tumor or lymphoma) must be considered.

In all patients a full clinical history (duration/intensity of complaints, time points of pain during the day, prior benign and malignant lesions, family history, and previous radiotherapy) and examination (with specific attention to size/consistency of swelling, location and relation of swelling to bone, demarcation and mobility of swelling and palpation of regional, and local lymph nodes) should be performed, taking into account the most likely diagnoses listed above. In patients under the age of 40 , investigations prior to referral should include X-ray of the affected bone and chest as well as simple blood tests [(full blood count (FBC), erythrocyte sedimentation rate (ESR), biochemical profile including alkaline phosphatase (ALP)]. Magnetic resonance imaging (MRI) can be done prior to or after referral [15].

In patients over the age of 40 , where metastatic disease is more common, patients should be more extensively investigated to exclude a primary tumour at another location, ideally with computed tomography (CT) of the chest, abdomen and pelvis, isotope bone scan, and a myeloma screen prior to referral-provided these can be done quickly. If the lesion is solitary and no primary site identified, then the patient should be referred to one of the reference centres.

All patients with clinicoradiological findings suggestive of a primary bone tumour should be sent to a bone tumour reference centre prior to biopsy. In England, there are five designated centres.

\subsection{UK Reference Centres.}

(i) Royal National Orthopaedic Hospital: London, phone 020 8909 5584/5600, fax 0208909 5709, http:// www.londonsarcoma.org.

(ii) Royal Orthopaedic Hospital: Birmingham, phone 0121685 4150, Fax 01216854146.

(iii) Nuffield Orthopaedic Centre: Oxford, phone 01865 738061.

(iv) North of England Bone and Soft Tissue Tumour Service, Freeman Hospital: Newcastle upon Tyne phone 0191223 1496, fax 0191233 1328, http://www .newcastlesarcoma.org.uk.

(v) Greater Manchester and Oswestry Sarcoma Service, Robert Jones and Agnes Hunt Hospital: Oswestry, phone 08458383429 , fax 08458383428 .

3.3. Management at Reference Centre. All patients with a suspected bone sarcoma should be referred for diagnosis and have their care managed at a reference centre by a fully accredited multi disciplinary team (MDT) $[31,32]$. This is a core principle of the NICE Guidance "improving outcomes for patients with sarcomas" [8] and "improving outcomes with children and young people with cancer" [33]. This guidance also recommends the following.

(i) Networks should ensure that GPs are aware of and comply with the urgent referral criteria in the NICE "referral guidelines for suspected cancer" and that GPs and hospital doctors are aware of the diagnostic pathways.

(ii) All patients with a confirmed diagnosis of bone sarcoma should have their care supervised by or in conjunction with a sarcoma MDT and be allocated a key worker. Children, teenagers, and young adults should also be discussed at the relevant children's or TYA (young adult) MDT.

(a) Networks should ensure that they meet the needs of children and young people with cancer with sufficient specialist staff and care and facilities appropriate to the child or young persons age.

(iii) A bone sarcoma MDT should meet minimum criteria for the number of patients treated in each year and adhere to the requirements for core membership of the relevant specialties.

(a) All sarcoma MDTs should collect data on patients, tumours, treatment, and outcomes as agreed nationally.

(iv) All patients with a provisional histological and/or radiological diagnosis of bone sarcoma should have their diagnosis reviewed by a specialist sarcoma pathologist and/or radiologist who are part of a sarcoma MDT. 
(v) Patients should undergo definitive resection of their sarcoma by a surgeon who is a member of a sarcoma MDT or by a surgeon with tumour site-specific or age-appropriate skills, in consultation with the sarcoma MDT.

(vi) Chemotherapy and radiotherapy are important components of the treatment of some patients and should be carried out at designated centres by appropriate specialists as recommended by a sarcoma MDT.

(vii) Patients should be informed about relevant clinical trials and supported to enter them.

Furthermore, referral of patients to the reference centre for initial biopsy is also strongly recommended. This obviates the risk associated with improperly performed biopsies and increases the proportion of patients whose tumours can be studied with modern molecular techniques.

\section{Investigation}

4.1. Imaging. All patients should have plain X-rays in two planes at presentation. CT should only be used in cases where there is diagnostic uncertainty and to optimally visualise areas of microcalcification, periosteal bone formation, cortical destruction, or soft tissue involvement. CT can also be helpful for surgical planning in the pelvic area. When the diagnosis of malignancy is possible on radiographs, MRI of the whole bone is the next most appropriate imaging investigation and is the best modality for local staging [34].

General staging (where indicated) to assess the extent of distant disease should include chest radiography and/or CT. CT remains the diagnostic technique of choice for imaging the chest [35], (appreciating that small nodules are not specific for malignancy). Whole body bone scintigraphy will detect lesions elsewhere in the skeleton [3]. Whole body MRI and positron emission tomography (PET) are under evaluation both for staging and treatment response evaluation [36]. Additional appropriate imaging studies and biopsies should be taken of suspicious sites, as accurate staging of the disease has an impact on treatment and outcome. Guidance on the management of small suspicious lung nodules is available in trial protocols $[37,38]$.

In the case of chondrosarcoma, a contrast enhanced MRI can reveal high-grade areas, which is useful to guide the site of biopsy.

4.2. Staging. There are two common staging systems in use, the Enneking [39] and the TNM system (American Joint Committee on Cancer-AJCC/International Union against cancer-UICC) [40].

The Enneking system is based on tumour grade ( $\mathrm{I}=$ low and II = high grade) and extent in relation to the anatomical compartments of the limb. Where the bone cortex is intact, the tumour is deemed to be intracompartmental. Stage III tumours can be any grade with metastases. This is summarized in Table 2.

The TNM (AJCC/UICC) system is based on tumour grade, size, and the presence of metastases (Table 3 ).
4.3. Laboratory Tests. No specific laboratory tests for diagnosis of bone sarcoma are available. However, some may be of prognostic value; examples include ESR, alkaline phosphatase (ALP) and lactate dehydrogenase (LDH) [41, 42].

4.4. Other Baseline Assessments. Chemotherapy treatment can result in renal, cardiac, and auditory dysfunction [43], and patients undergoing this treatment must have baseline renal function testing and assessment of cardiac function as well as an audiogram (in case of treatment with cisplatin). Sperm storage is recommended for male patients of reproductive age. For female patients, a fertility physician should be consulted to discuss options with the patient.

4.5. Biopsy. The definitive test remains biopsy. The biopsy of a suspected primary malignant bone tumour should be carried out at the reference centre by the surgical team who are to carry out the definitive tumour resection. Inappropriate techniques can irrevocably compromise the chance of limb salvage or even cure. The principles of biopsy [11] are the following:

(i) Biopsy should only be done after local imaging of the affected bone to allow planning of the most representative area to biopsy.

(ii) There should be minimal contamination of normal tissues.

(iii) In many situations, core needle biopsy will be adequate, often controlled by ultrasound, X-ray or CT.

(iv) Samples should always be taken for micro biological culture as well as histology and cytogenetic studies.

(v) In the reference centre, samples should ideally be snap frozen for storage in a tumour bank for future translational research studies (patient consent should be obtained).

(vi) Samples must be interpreted by an experienced pathologist.

(vii) The request form should contain sufficient detail for a pathologist to make a diagnosis, including the site of the tumour, the patient's age, and the radiological differential diagnosis.

CT guided biopsies [44, 45] are appropriate for deeper locations (e.g., pelvis) or to target a particular area of concern (e.g., a possibly dedifferentiated area in a chondrosarcoma). In cases of uncertainty that the tissue is representative, frozen section may be considered in case more material is required. In aggressive and malignant tumours of bone, the biopsy track should be considered to be contaminated with tumour and must be removed together with the resection specimen at definitive surgery to avoid local recurrence; this includes possible channels through which drains have been placed. Biopsy tracks should be clearly marked by means of a small incision to ensure that the location can be recognised at the definitive procedure. 
TABLE 2: Enneking staging.

\begin{tabular}{lccc}
\hline Stage & Grade & Tumour & Metastasis \\
\hline IA & G1 low grade & T1 cortex intact (intracompartmental) & M0 \\
IB & G1 low grade & T2 cortex breached with soft tissue extension & M0 \\
IIA & G2 high grade & T1 cortex intact & M0 \\
IIB & G2 high grade & T2 cortex breached with soft tissue extension & M0 \\
IIIA & G1 or G2 & T1 & M1 \\
IIIB & G1 or G2 & T2 & M1 \\
\hline
\end{tabular}

TABle 3: AJCC/UICC staging.

\begin{tabular}{lccrc}
\hline Stage & Tumour $(\mathrm{T})$ & Node $(\mathrm{N})$ & Metastasis $(\mathrm{M})$ & Grade $(\mathrm{G})$ \\
\hline Stage IA & T1 (tumour $8 \mathrm{~cm}$ or less) & No & M0 & G1, 2 low grade \\
Stage IB & T2 (tumour more than $8 \mathrm{~cm})$ & No & M1, 2 low grade \\
Stage IIA & T1 & No & G3, 4 high grade \\
Stage IIB & T2 & No & M0 & G3, 4 high grade \\
Stage III & T3 (discontinuous tumours in primary site) & No & Any G \\
Stage IVA & Any T & N1 (regional lymph nodes) & Any M & Any G \\
Stage IVB & Any T & Any N & Any G (other sites) & Any G \\
\hline
\end{tabular}

Biopsy of other indeterminate lesions should always be considered if it will affect patient management (e.g., entry into a trial or local control decisions).

In cases of spinal column involvement, laminectomy or decompression should be avoided unless necessary to relieve spinal cord compression, and only after consultation with members of the bone sarcoma MDT.

4.6. Pathology. The pathologist reporting on both the diagnosis and resection of bone sarcomas should be an accredited bone tumour pathologist and part of a bone sarcoma MDT. The report should comply with guidance from the Royal College of Pathologists [46].

The biopsy report should include a description of the specimen received and the microscopic findings including the histological diagnosis. Histopathology reports of resection specimens should include a gross specimen description which records the location and size (measured in three dimensions in $\mathrm{mm}$ ) of the tumour in the resected bone. The histological features of the tumour should be described and the tumour type (and subtype) classified according to the latest WHO criteria [16]. The pathology report should note the extent of local tumour spread, including involvement of specific anatomical compartments. Whether the resection margins are clear or involved should be noted and the distance (in $\mathrm{mm}$ ) of infiltrating tumour from the nearest resection margin and the nature of tissue at this resection margin specified. Results of relevant ancillary investigations (e.g. immunohistochemistry or molecular genetics) should be recorded and the tumour should be coded using the
Systematized Nomenclature of Medicine-Clinical Terms (SNOMED-CT) codes [47].

The extent of tumor necrosis in response to any preoperative therapy should be assessed as being greater or less than $90 \%$ necrosis in broad terms although much greater specificity can be obtained.

4.7. Molecular Genetics and Pathology. Tumour banks are instrumental for diagnostic and translational research in the molecular pathology of cancer; therefore, informed consent for tumour banking should be sought that allows for later analysis and research according to local practice. In specialist centres, storage of fresh frozen tissue should be undertaken in every case where consent has been given.

Although most Ewing sarcomas can be recognised morphologically and by immunohistochemistry for the surface glycoprotein CD99, Ewing sarcoma translocation detection is mandatory when the clinicopathological presentation is unusual or the histological diagnosis is doubtful. A reference laboratory for Ewing sarcoma diagnosis should have both interphase fluorescence in situ hybridisation (FISH) and reverse transcription-polymerase chain reaction (RT-PCR) technology available [48]. The laboratory should be a participant in an external quality assurance programme.

4.8. Confirmation of Diagnosis. In every case, the diagnosis must be confirmed by reference to clinical findings, laboratory investigation and, in particular, radiological imaging at a sarcoma MDT. Ideally, all patients with suspected bone tumours should be discussed at the MDT meeting with the 
surgeon, the radiologist who has interpreted the imaging and the pathologist who has reviewed the biopsy material and an oncologist [8]; this will minimise the risk of errors in diagnosis and management.

\section{Prognostic Factors}

5.1. Osteosarcoma. Estimates of patients with newly diagnosed osteosarcoma with metastases, mainly located in the lung $[14,49,50]$, vary from $12.4 \%-34.4 \%$ rising to $40 \%$ of all cases over time [51]. Microscopic, subclinical metastatic disease is present in the majority of patients at diagnosis. Adverse prognostic or predictive factors include detectable primary metastases as well as axial or proximal extremity tumour site, large tumour volume, elevated serum alkaline phosphatase or LDH, older age, and poor histological response to preoperative chemotherapy/level of chemotherapy induced necrosis $[41,52]$.

5.2. Ewing Sarcoma. Around $26 \%$ of patients are diagnosed with metastatic disease (10\% lung, 10\% bones/bone marrow, $6 \%$ combinations, or others) [25]. Bone metastases confer a poorer outcome than lung/pleura metastases ( $<21 \%$ compared with 55\% 5-year relapse free survival) [53]. Other known prognostic factors are tumour site/axial location or volume, raised serum LDH levels and older age ( $>15$ years). A poor histological response to preoperative chemotherapy and incomplete or no surgery for local therapy are further adverse prognostic factors $[42,52,54,55]$.

5.3. Chondrosarcoma. All chondrosarcomas have a significant risk of local recurrence, but metastases rarely arise in patients with grade I tumours. Grade II and III chondrosarcomas are often grouped together even though there is a wide spectrum of outcome-grade and stage are independent prognostic factors [56] along with histology [27]. Clear cell chondrosarcoma behaves like a low-grade chondrosarcoma, whilst mesenchymal chondrosarcoma should be considered a high-grade bone sarcoma. Dedifferentiated chondrosarcomas are high-grade aggressive sarcomas and frequently metastasise to lungs and other bones $[26,30]$.

5.4. Spindle Cell Sarcomas of Bone. Prognosis is similar to patients with osteosarcoma of the same age and is related to the same prognostic factors.

\section{Management Overview}

Bone sarcoma is a potentially curable disease with surgery and chemotherapy being the mainstays of treatmentnotably preoperative "neoadjuvant" systemic combination chemotherapy, local surgery, and postoperative "adjuvant" chemotherapy [43]. One of the main goals is to decrease the incidence of a subsequent distant relapse [57]. Despite a lack of new treatments over time, outcomes have improved, due to use of more aggressive multimodal treatments and utilisation of surgery for sites previously considered inoperable [58].
Survival for patients with bone tumours has improved substantially over the last 30 years in the UK. A national study looking at the survival of patients aged 15 years or older with bone cancer of all types found that 5-year relative survival rates increased from $29 \%$ in $1971-1975$ to $51 \%$ in $1986-1990$ [59]. Patterns of survival for patients under 40 in the UK showed an increase from $42 \%$ to $53 \%$ in the period from the early ' 80 s to the early ' 90 s for osteosarcoma and $31 \%$ to $51 \%$ for Ewing sarcoma [60]. The EUROCARE data for children aged $0-14$ years showed an increase from $52 \%$ to $60 \%$ for osteosarcoma, and $50 \%$ to $60 \%$ for Ewing sarcoma during the latter 4 years of a survey performed between 1978 and 1989 when compared to the entire 11 year period [10]. Data from northern England and the West Midlands for the period 1981-2002 showed an improvement for Ewing sarcoma but not for osteosarcoma for a number of reasons including potential delays in diagnosis, accrual to trials, adherence to therapy and lack of improvement in treatment strategies [17].

6.1. Systemic Chemotherapy. As malignant primary bone tumours are rare and management is complex, the accepted standard is treatment in reference centres or within reference networks able to provide access to the full spectrum of care in predetermined partnerships with such centres $[2,8]$.

Therapy is usually given within the framework of prospective, often collaborative, clinical studies, or established treatment protocols. In cases of high-grade osteosarcoma, Ewing sarcoma or spindle cell sarcoma (neo) adjuvant therapy is indicated, preferably within the framework of international clinical trials.

6.2. Surgery. Surgery of the primary tumour should be performed only after adequate preoperative staging, striving to obtain adequate surgical margins. A wide enbloc resection of the affected part of the bone and soft tissue should be performed. Close surgical margins may be identified with (MRI-inert) haemoclips.

Decisions about the optimal surgical procedure (i.e., limb salvage or amputation) should be made as part of an MDT discussion, taking into account tumour and treatment factors (e.g., extent of tumour and response to neoadjuvant therapy). The type of surgical reconstruction will depend on patient and surgeon choice and experience following open discussion of the risks and benefits of different options. Surgical excision of local recurrence or metastatic disease should usually be considered following decisions being made by the MDT.

6.2.1. Requirements for the Surgical Report. The surgeon should describe the surgical procedure carried out and indicate the tissues resected. The planned surgical margin should be identified along with any areas of concern. The type of reconstruction should be described and the postoperative care to be used. The use of prophylactic antibiotics and anticoagulants should be clearly stated. The specimen should be orientated to allow the pathologist to adequately describe the anatomical location of close surgical margins. 
6.3. Radiotherapy. The role of radiotherapy in osteosarcoma and chondrosarcoma is limited, as these tumours are recognised as being relatively radioresistant. Thus, radiotherapy is only used as definitive treatment of a primary tumour if there is no surgical option. It can be usefully used to achieve local tumour control at least in the short to mid-term although it is unlikely to achieve long term tumour control. However, there is limited evidence that osteosarcoma can sometimes be controlled by radiotherapy alone if there has been a good subjective response to chemotherapy [61]. If radiotherapy is to be used as definitive local treatment, a radical dose of radiotherapy is required. Standard conformal radiotherapy may not be able to achieve an adequate radiotherapy dose to the tumour, in which case techniques such as IMRT (intensity modulated radiotherapy) may allow delivery of a higher radiotherapy dose [62]. In addition, insertion of pelvic spacer devices can enable displacement of bowel away from pelvic tumours, which can also facilitate delivery of a higher dose. Heavy particle therapy with protons or carbon ions, often in combination with photons, are being used increasingly in the treatment of primary bone sarcomas not amenable to surgery [63-65]. Excellent outcomes have been reported for skull base chondrosarcomas with proton beam radiotherapy achieving approximately 70\%-90\% local control rates when combined with surgery [66], and the reported early results in bone sarcomas are encouraging. However, this approach is still relatively new and is considered at present as exploratory. At present, there is no proton facility in the United Kingdom, but potentially suitable cases can be submitted to the UK Proton Panel for consideration for approval for funding for treatment at a facility overseas.

Radiotherapy may be used postoperatively in osteosarcoma and chondrosarcoma in selected cases, if there are concerns regarding (usually soft tissue) resections margins, or possible soft tissue contamination, and if further surgery is not possible [63].

In contrast with osteosarcoma and chondrosarcoma, Ewing Sarcoma is undoubtedly a radiation-sensitive tumour, and as such, radiotherapy can be usefully utilised as part of management. It may be used as definitive local therapy if surgery is not possible, with curative intent. It may also be used in combination with surgery, for patients who have had a poor histological response to chemotherapy, or when there are concerns regarding surgical resection margins $[67,68]$. There is also a potential role for whole lung radiotherapy (WLRT) for patients with lung metastases [69, 70], and indeed, WLRT is included as part of treatment for patients with lung metastases in the current Euro-Ewing-99 protocol.

Radiotherapy can be used for all bone sarcoma types for palliation of metastatic disease.

6.4. Prevention and Management of Pathological Fracture. Where there is an existing pathological fracture in a possibly malignant primary bone tumour, adequate imaging should be performed including MRI followed by biopsy. In cases of fracture, internal fixation is contraindicated as it disseminates tumour further into both bone and soft tissues and increases the risk of local recurrence. External splintage is recommended, along with appropriate pain control. In patients with weakened bone apparent at presentation, there may also be a strong case for immobilising the part after biopsy.

Neoadjuvant chemotherapy should be used in the expectation that a good response will allow the fracture haematoma to contract and allow subsequent resection of the tumour and the involved soft tissues. In patients with a poor response to chemotherapy, or tumours unlikely to respond to chemotherapy then early surgery obtaining wide margins should be considered, which may require amputation. Postoperative radiotherapy may be considered to try to decrease the risk of local recurrence [71].

\section{Specific Treatment}

\subsection{Osteosarcoma}

7.1.1. Localised Disease. Curative treatment for high-grade osteosarcoma consists of surgery and chemotherapy [2, 43]. Compared with surgery alone, multimodal treatment of high-grade osteosarcoma increases survival probabilities from only $10 \%-20 \%$ to around $60 \%$ [72].

The goal of surgery is to safely remove the tumour and yet preserve as much function as possible. Most patients should be considered candidates for limb salvage. Wide surgical margins should be planned accepting that an apparent good response of the tumour to chemotherapy may allow a closer margin of excision to be considered safe.

Doxorubicin, cisplatin, high-dose methotrexate, and ifosfamide have demonstrated antitumour activity in osteosarcoma [31, 72-75] and are usually administered in protocols involving 3 or 4 drug combinations. These drugs should be administered with adequate supportive care by experienced paediatric oncologists or medical oncologists in reference institutions with appropriate infrastructure with a MDT approach [43]. A variety of pre- and postoperative combinations are used in common practice and in clinical trials, and the ideal combination regimen and the optimal treatment duration are yet to be defined or confirmed $[2,76]$.

Most current protocols include a period of preoperative chemotherapy. This has not been proven to add survival benefit over postoperative chemotherapy alone, although there are clear practical advantages [31, 77]. Treatment is commonly given over periods of 6-9 months. The extent of histological response to pre operative chemotherapy however offers important prognostic information [78], but altering postoperative chemotherapy on the basis of response is not recommended outside of ongoing trials [38], and it is not accepted as a reliable surrogate endpoint for overall outcome $[43,72]$. The use of haematopoietic growth factors to increase dose intensity has not consistently resulted in improved survival of osteosarcoma patients [72] but may limit morbidity associated with myelosuppression.

The immune modulator liposomal muramyl tripeptide (mifamurtide) added to postoperative chemotherapy demonstrated a statistically significant advantage in overall survival and a trend in event-free survival in a large randomised trial [79] and has been approved in Europe 
for patients under 30 with completely resected localised osteosarcoma.

Whenever possible, patients with osteosarcoma should receive chemotherapy in the context of prospective trials, which is regarded as standard of care. Chemotherapy is also recommended for older patients with osteosarcoma using adapted protocols [20].

Low-grade central and parosteal osteosarcoma are variants with lower malignant potential, which are treated by surgery only. Careful analysis of the resected tumour may show areas of high-grade change in which case the patient should be treated as for a conventional osteosarcoma. The exact role of chemotherapy has not been defined for periosteal and jaw osteosarcoma, but experience has shown that chemotherapy can be given as standard. Jaw and other craniofacial osteosarcomas present specific problems for management, especially to achieve local control and should always be referred to a reference centre and discussed at a sarcoma MDT before surgical intervention.

7.1.2. Metastatic and Recurrent Disease. Patients presenting with metastatic osteosarcoma are a heterogeneous group and may be treated using the same regimens used for nonmetastatic osteosarcomas provided that surgical resection of all sites of disease is deemed feasible [80]. Approximately $30 \%$ of all patients with primary metastatic osteosarcoma and $>40 \%$ of those who achieve a complete surgical remission become long-term survivors [2].

The management of recurrent osteosarcoma needs to take into account the timing of recurrence, the site of recurrence, and the number of metastases. Treatment for recurrent osteosarcoma is primarily surgical, be it local recurrence or metastatic. Prognosis is poor, with long-term postrelapse survival of less than a third [2].

Pulmonary metastasectomy can play a major role but only if complete removal of all metastases can be achieved [51], as the disease is otherwise almost universally fatal. More than a third of patients with a second surgical remission survive for $>5$ years, even patients with multiple recurrences may be cured as long as recurrences are resectable, and repeated thoracotomies are often warranted [81].

The role of second-line chemotherapy for recurrent osteosarcoma is less well defined than that of surgery, and there is no accepted standard regimen $[2,81]$. The choice of agents may take into account the prior disease-free interval and often includes ifosfamide \pm etoposide, or possibly docetaxel/gemcitabine. The use of second-line chemotherapy has been shown to correlate with limited prolongation of survival in patients with inoperable metastatic recurrences, and a positive correlation in operable disease was observed in one series [82-84]. Radiotherapy to inoperable sites may be indicated for palliation.

\subsection{Ewing Sarcoma}

7.2.1. Localised Disease. With surgery or radiotherapy alone, 5 -year survival is $<10 \%$. With treatment in current multimodality trials including chemotherapy, 5 -year survival is $\sim 60 \%-70 \%$ in localised and $\sim 20 \%-40 \%$ in metastatic disease [3].

All current trials employ three to six cycles of initial chemotherapy after biopsy, followed by local therapy and another six to ten cycles of chemotherapy usually given at 2 or 3 week intervals based on current agreed national or international protocols. Treatment duration is thus 10-12 months. Agents considered most active include doxorubicin, cyclophosphamide, ifosfamide, vincristine, dactinomycin, and etoposide $[25,37,52,54,68,69,85-90]$. Virtually all active protocols are based on four to six drug combinations of these agents. Chemotherapy intensity is positively associated with outcome. High-dose chemotherapy with blood stem cell transplantation is still investigational.

Despite lively debate, complete surgery, where feasible, is regarded as the best modality of local control given the higher risk of local recurrence when radiotherapy is used as sole treatment for the primary tumour. Radiotherapy alone should be considered if complete surgery is impossible or if it will be very disabling. Individual decisions about local therapy are frequently complex and should only be made by an experienced reference centre MDT in conjunction with the parents and family.

Postoperative radiotherapy should be given in cases of inadequate surgical margins and discussed where histological response in the surgical specimen was poor (i.e., $>10 \%$ viable tumour cells). Tolerability of therapies in adults needs to be taken into account when transferring treatment protocols conceived for children and adults $\leq 40$ years.

7.2.2. Metastatic and Recurrent Disease. Patients with metastases at diagnosis are treated similarly to patients with localised disease but have a worse prognosis. Several nonrandomised trials have assessed the value of more intensive, time compressed or high-dose chemotherapy approaches, followed by autologous stem cell rescue, but evidence of benefit, resulting from trials, is pending [70, 91, 92]. In patients with lung metastases, whole lung irradiation may confer a survival advantage but firm data are lacking and a systematic review failed to confirm a survival advantage [93]. The role of surgical resection of residual metastases is less well defined. Patients with bone or bone marrow metastases and patients with recurrent disease still fare poorly, with 5year survival rates of $\sim 20 \%[3,94]$.

Prognostic factors relate to site of and time to relapse: patients relapsing later than 2 years from initial diagnosis and with disease not involving bone marrow or multiple bones have a better outcome $[95,96]$. Doxorubicin therapy is usually no longer feasible due to previously achieved cumulative doses. Chemotherapy regimens in relapse situations are not standardised and are currently often based on alkylating agents (cyclophosphamide, high-dose ifosfamide) in combination with topoisomerase inhibitors (etoposide, topotecan) or irinotecan with temozolomide. $[85,90]$.

Radiotherapy may be helpful to palliate local symptoms.

7.3. Chondrosarcoma. Assessing the grade of chondrosarcomas is difficult and variations in opinions, even between 
experts are common [28]. Low-grade cartilage tumours are unlikely to metastasise but may recur locally. Biopsy confirmed low grade central chondrosarcomas in the long bones of the limbs can be managed by curettage with or without adjuvant (e.g., phenol, cement, and cryotherapy) with a high chance of success. Low-grade peripheral chondrosarcomas (arising from osteochondromas) should be surgically excised, aiming to excise the tumour with a covering of normal tissue over it. Higher-grade chondrosarcomas (including clear cell chondrosarcoma), and all chondrosarcomas of the pelvis or axial skeleton should be surgically excised with wide margins $[26,29]$.

Recent evidence suggests that mesenchymal chondrosarcoma may be responsive to chemotherapy and may be considered for adjuvant or neoadjuvant therapy [97, 98]. There remains uncertainty about chemotherapy sensitivity of de-differentiated chondrosarcoma but it is often treated like osteosarcoma, with poorer outcome [30]. There is a very high risk of local recurrence following excision of dedifferentiated chondrosarcoma, particularly in the presence of a pathological fracture. If wide margins cannot be reliably achieved with limb salvage then amputation may be considered but the metastases remain a problem.

7.4. Spindle Cell Sarcomas of Bone. Treatment strategies mimic those of osteosarcoma, with age-adjusted chemotherapy and complete enbloc resection including any soft-tissue component.

7.5. Other Bone Sarcomas. Although conventional therapy for chordomas has in the past been complete surgical resection [99], there are encouraging results from high-dose radiotherapy using proton beams or carbon ion facilities $[100,101]$. Assessment in a specialist centre with expertise in managing these tumours is essential to define the role of surgery and/or radiotherapy. Metastases are rare but local recurrence common. There is evidence of some effectiveness of molecular targeted agents [102].

Most adamantinomas are low-grade but will recur if not completely resected. Higher-grade areas either in the primary tumour or in the recurrence may require systemic therapy.

\section{Treatment Evaluation}

Imaging whilst the patient is on chemotherapy is limited to intermittent assessment of the primary and metastatic sites of bone disease by clinical means (pain and clinical measurement) conventional radiographs, and assessment of the lungs by CT [34].

8.1. Osteosarcoma. Change in the size and ossification of the tumour is not a reliable guide to tumour response to neoadjuvant chemotherapy. Assessment of MRI detected peritumoural oedema is helpful: its disappearance is a sign of a good treatment response [103]. Dynamic MRI is reliable, but requires sequential scans to evaluate change in tumour vascularity [104]. Assessment of response is usually only apparent after several cycles of chemotherapy.
8.2. Ewing Sarcoma. Change in the size of the soft tissue mass is easily evaluated on MRI and is a reliable indicator of tumour response [105]. Dynamic MRI is not as reliable as in osteosarcoma, as remaining small tumour foci may not be detected. Sequential fluorodeoxyglucose PET (FDG PET) evaluation may be of additional value [34].

Progressive disease whilst on chemotherapy may mandate changes in treatment or earlier primary local control measures. An increase in the size of a tumour may, however, be due to necrosis rather than tumour progression.

\section{Followup}

Followup is designed to detect either local recurrence or metastatic disease at a time when early treatment is still possible and might be effective [34]. Followup of high grade tumours should include both a physical examination of the tumour site and assessment of the function and possible complications of any reconstruction. Local and chest imaging should be the norm. Evidence of optimum frequency of followup and the best imaging investigations is still lacking [34].

For high-grade tumors, current protocols recommend follow up intervals of $2-4$ months for the first 3 years after completion of therapy, every 6 months for year 4 and 5 and thereafter annually $[2,3]$.

For low-grade bone sarcomas, the frequency of followup visits may be reduced to $4-6$ monthly for 2 years and then annually. Late metastases as well as local recurrences and functional deficits may occur $>10$ years after diagnosis in all tumours, and there is no universally accepted stopping point for tumour surveillance [56].

It is important to evaluate the long-term toxicity effect of chemotherapy and radiotherapy as well as immediate chemotherapy related complications [43]. Monitoring for late effects should be undertaken, depending on the chemotherapy protocol and radiation used and in conjunction with late effect services when available $[54,106,107]$.

Secondary cancers may arise in survivors of bone sarcomas, either related to or independent of irradiation. Secondary leukaemia, particularly acute myeloid leukaemia, may rarely be observed following chemotherapy as early as $2-5$ years after treatment $[108,109]$.

\section{Key Recommendations}

(i) The most common symptom of a primary bone tumour is pain which may be non mechanical or night pain. The presence of bone pain at night should always be considered to be a "red flag" symptom leading to further investigation. The presence of pain or a palpable mass arising from any bone should cause concern and lead to further investigation of which a plain X-ray is the first investigation of choice.

(ii) The presence of radiological features including bone destruction, new bone formation, periosteal swelling, and soft-tissue swelling are suggestive, but not diagnostic of a bone tumour and should also lead to further investigation. 
(iii) All bone tumour patients with clinicoradiological findings suggestive of a primary bone tumour should be sent to a reference centre.

(iv) The definitive diagnostic test is a biopsy. The biopsy of a suspected primary malignant bone tumour should be carried out at the reference centre by the surgical team who are to carry out the definitive tumour resection.

(v) The pathologist reporting on both the diagnosis and resection of bone sarcomas should be an accredited bone tumour pathologist and part of a bone sarcoma multidisciplinary team (MDT). The report should comply with guidance from the Royal College of Pathologists.

(vi) In every case, the diagnosis must be confirmed by reference to clinical findings, laboratory investigation and, in particular, radiological imaging at a bone sarcoma MDT, this will minimise the risk of errors in diagnosis and management. All patients should have tissue stored for subsequent investigation with appropriate consent.

(vii) Patients should have their care managed at a reference centre by a fully accredited MDT. The MDT should make the decision about chemotherapy but may delegate the responsibility to another centre. All surgery should be carried out at a specialist bone sarcoma centre.

(viii) Curative treatment for high-grade osteosarcoma consists of surgery and chemotherapy. All patients should be considered for inclusion into national or international clinical trials.

(ix) Treatment for recurrent osteosarcoma is primarily surgical, be it local recurrence or metastatic. The role of second-line chemotherapy for recurrent osteosarcoma is less well defined than that of surgery and there is no accepted standard regimen.

(x) For Ewing sarcoma, systemic treatment with chemotherapy is standard. Where possible, surgery is preferred for local control over radiotherapy alone.

(xi) Management of chondrosarcoma is surgical excision with wide margins for all but low-grade central limb chondrosarcoma where curettage may be adequate. There are no data to support the routine use of chemotherapy.

(xii) Standard follow up for all sarcoma cases is currently chest X-ray and clinical review. The role of regularcross sectional imaging remains uncertain.

\section{References}

[1] National Comprehensive Cancer Network, "NCCN National Clinical Practice Guidelines in Oncology," Version 2, 2010, http://www.nccen.org/.

[2] S. Bielack, D. Carrle, and P. G. Casali, "Osteosarcoma: ESMO clinical recommendations for diagnosis, treatment and follow-up," Annals of Oncology, vol. 20, supplement 4, pp. iv137-iv139, 2009.
[3] M. Paulussen, S. Bielack, H. Jürgens, and P. G. Casali, "Ewing's sarcoma of the bone: ESMO clinical recommendations for diagnosis, treatment and follow-up," Annals of Oncology, vol. 20, supplement 4, pp. iv140-iv142, 2009.

[4] P. C. W. Hogendoorn, "Bone sarcomas: ESMO clinical recommendations for diagnosis, treatment and follow up," Annals of Oncology, vol. 21, supplement 5, pp. v200-v209, 2010.

[5] D. J. Cook, G. H. Guyatt, A. Laupacis et al., "Rules of evidence and clinical recommendations on the use of antithrombotic agents," Chest, vol. 102, no. 4, supplement, pp. 305S-311S, 1992.

[6] A. V. Maheshwari and E. Y. Cheng, "Ewing sarcoma family of tumours," Journal of American Academy of Orthopaedic Surgeons, vol. 18, no. 2, pp. 94-107, 2010.

[7] P. J. Messerschmitt, R. M. Garcia, F. W. Abdul-Karim, E. M. Greenfield, and P. J. Getty, "Osteosarcoma," Journal of the American Academy of Orthopaedic Surgeons, vol. 17, no. 8, pp. 515-527, 2009.

[8] National Institute for Health and Clinical Excellence (NICE), "Guidance on Cancer Services-Improving Outcomes for People with Sarcoma: the Manual [Needs assessment, Evidence review, List of recommendations]," 2006, (Developed by the National Collaborating Centre for Cancer).

[9] L. Mirabello, R. J. Troisi, and S. A. Savage, "International osteosarcoma incidence patterns in children and adolescents, middle ages and elderly persons," International Journal of Cancer, vol. 125, no. 1, pp. 229-234, 2009.

[10] C. A. Stiller, A. W. Craft, and I. Corazziari, "Survival of children with bone sarcoma in Europe since 1978: results from the EUROCARE study," European Journal of Cancer, vol. 37, no. 6, pp. 760-766, 2001.

[11] H. J. Mankin, T. A. Lange, and S. S. Spanier, "The hazards of biopsy in patients with malignant primary bone and softtissue tumors," Journal of Bone and Joint Surgery. Series A, vol. 64, no. 8, pp. 1121-1127, 1982.

[12] E. I. Hauben and P. C. W. Hogendoorn, "Epidemiology of primary bone tumors and economical aspects of bone metastases," in Bone Cancer. Progression and Therapeutic Approaches, D. Heymann, Ed., pp. 3-8, Academic Press, London, UK, 1st edition, 2009.

[13] R. J. Grimer and R. S. Sneath, "Diagnosing malignant bone tumours," Journal of Bone and Joint Surgery. Series B, vol. 72, no. 5, pp. 754-756, 1990.

[14] P. A. Pizzo and D. G. Poplack, Osteosarcoma. Principles and Practice of Pediatric Oncology, vol. 35, 5th edition, 2006.

[15] R. J. Grimer and T. R. Briggs, "Earlier diagnosis of bone and soft tissue tumours," Journal of Bone and Joint Surgery, vol. 92-B, no. 11, pp. 1489-1492, 2010.

[16] C. D. M. Fletcher, K. K. Unni, and F. Mertens, WHO Classification of Tumours. Pathology and Genetics of Tumours of Soft Tissue and Bone, IARC Press, Lyon, France, 2002.

[17] R. Eyre, R. G. Feltbower, E. Mubwandarikwa, T. O. B. Eden, and R. J. Q. McNally, "Epidemiology of bone tumours in children and young adults," Pediatric Blood and Cancer, vol. 53, no. 6, pp. 941-952, 2009.

[18] S. Kalra, R. J. Grimer, D. Spooner et al., "Radiation induced sarcomas of bone. Factors that affect outcome," The Journal of Bone and Joint Surgery, vol. 89, pp. 808-813, 2007.

[19] National Cancer Intelligence Network, 2010, http://www .ncin.org.uk/.

[20] R. J. Grimer, S. R. Cannon, A. M. Taminiau et al., "Osteosarcoma over the age of forty," European Journal of Cancer, vol. 39, no. 2, pp. 157-163, 2003. 
[21] H. D. Dorfman and B. Czerniak, "Bone cancers," Cancer, vol. 75, no. 1, pp. 203-210, 1995.

[22] L. Mascarenhas, S. Siegel, L. Spector et al., "Malignant bone tumours," SEER AYA Monograph, vol. 8, pp. 97-109, 2002.

[23] B. Fuchs and D. J. Pritchard, "Etiology of osteosarcoma," Clinical Orthopaedics and Related Research, no. 397, pp. 4052, 2002.

[24] M. F. Hansen, M. Seton, and A. Merchant, "Osteosarcoma in Paget's disease of bone," Journal of Bone and Mineral Research, vol. 21, supplement, pp. P58-P63, 2006.

[25] M. Bernstein, H. Kovar, M. Paulussen et al., "Ewing's sarcoma family of tumors: current management," Oncologist, vol. 11, no. 5, pp. 503-519, 2006.

[26] R. F. Riedel, N. Larrier, L. Dodd, D. Kirsch, S. Martinez, and B. E. Brigman, "The clinical management of chondrosarcoma," Current Treatment Options in Oncology, vol. 10, no. 1-2, pp. 94-106, 2009.

[27] J. V. M. G. Bovée, A.-M. Cleton-Jansen, A. H. M. Taminiau, and P. C. W. Hogendoorn, "Emerging pathways in the development of chondrosarcoma of bone and implications for targeted treatment," Lancet Oncology, vol. 6, no. 8, pp. 599-607, 2005.

[28] D. Eefting, Y. M. Schrage, M. J. A. Geirnaerdt et al., "Assessment of interobserver variability and histologic parameters to improve reliability in classification and grading of central cartilaginous tumors," American Journal of Surgical Pathology, vol. 33, no. 1, pp. 50-57, 2009.

[29] H. Gelderblom, P. C. W. Hogendoorn, S. D. Dijkstra et al., "The clinical approach towards chondrosarcoma," Oncologist, vol. 13, no. 3, pp. 320-329, 2008.

[30] R. J. Grimer, G. Gosheger, A. Taminiau et al., "Dedifferentiated chondrosarcoma: prognostic factors and outcome from a European group," European Journal of Cancer, vol. 43, no. 14, pp. 2060-2065, 2007.

[31] S. S. Bielack, J.-N. Machatschek, S. Flege, and H. Jürgens, "Delaying surgery with chemotherapy for osteosarcoma of the extremities," Expert Opinion on Pharmacotherapy, vol. 5, no. 6, pp. 1243-1256, 2004.

[32] N. Federman, N. Bernthal, F. C. Eilber, and W. D. Tap, “The multidisciplinary management of osteosarcoma," Current Treatment Options in Oncology, vol. 10, no. 1-2, pp. 82-93, 2009.

[33] National Institute for Health and Clinical Excellence (NICE), "Improving outcomes with children and young people with cancer," 2005, http://guidance.nice.org.uk/CSGCYP.

[34] J. S. Meyer, H. R. Nadel, N. Marina et al., "Imaging guidelines for children with Ewing sarcoma and osteosarcoma: a report from the Children's Oncology Group Bone Tumor Committee," Pediatric Blood and Cancer, vol. 51, no. 2, pp. 163-170, 2008.

[35] P. Picci, D. Vanel, A. Briccoli et al., "Computed tomography of pulmonary metastases from osteosarcoma: the less poor technique. A study of 51 patients with histological correlation," Annals of Oncology, vol. 12, no. 11, pp. 1601-1604, 2001.

[36] M. R. Benz, N. Tchekmedyian, F. C. Eilber, N. Federman, J. Czernin, and W. D. Tap, "Utilization of positron emission tomography in the management of patients with sarcoma," Current Opinion in Oncology, vol. 21, no. 4, pp. 345-351, 2009.

[37] EURO-E.W.I.N.G 99 treatment manual, 2010, http://euroewing.klinikum.uni-muenster.de/.

[38] EURAMOS, 2010, http://www.ctu.mrc.ac.uk/euramos/.
[39] W. F. Enneking, S. S. Spanier, and M. Goodman, "A system for the surgical staging of musculoskeletal sarcoma," Clinical Orthopaedics and Related Research, vol. 153, pp. 106-120, 1980.

[40] AJCC Cancer Staging Manual, Springer, New York, NY, USA, 6th edition, 2002.

[41] J. A. M. Bramer, J. H. van Linge, R. J. Grimer, and R. J. P. M. Scholten, "Prognostic factors in localized extremity osteosarcoma: a systematic review," European Journal of Surgical Oncology, vol. 35, no. 10, pp. 1030-1036, 2009.

[42] P. J. Leavey and A. B. Collier, "Ewing sarcoma: prognostic criteria, outcomes and future treatment," Expert Review of Anticancer Therapy, vol. 8, no. 4, pp. 617-624, 2008.

[43] D. Carrle and S. S. Bielack, "Current strategies of chemotherapy in osteosarcoma," International Orthopaedics, vol. 30, no. 6, pp. 445-451, 2006.

[44] A. O. Altuntas, J. Slavin, P. J. Smith et al., "Accuracy of computed tomography guided core needle biopsy of musculoskeletal tumours," ANZ Journal of Surgery, vol. 75, no. 4, pp. 187-191, 2005.

[45] C. S. Pramesh, M. S. Deshpande, D. N. Pardiwala, M. G. Agarwal, and A. Puri, "Core needle biopsy for bone tumours," European Journal of Surgical Oncology, vol. 27, no. 7, pp. 668-671, 2001.

[46] Royal College of Pathologists Standards and datasets for reporting cancers: dataset for histopathology reports on primary bone tumours, 2010, http://www.rcpath.org/.

[47] SNOMED CT-the language of the NHS Care Records Service, 2007, http://www.connectingforhealth.nhs.uk/publications 3769.

[48] I. MacHado, R. Noguera, A. Pellin et al., "Molecular diagnosis of ewing sarcoma family of tumors: a comparative analysis of 560 cases with fish and rt-pcr," Diagnostic Molecular Pathology, vol. 18, no. 4, pp. 189-199, 2009.

[49] A. H. Aljubran, A. Griffin, M. Pintilie, and M. Blackstein, "Osteosarcoma in adolescents and adults: survival analysis with and without lung metastases," Annals of Oncology, vol. 20, no. 6, pp. 1136-1141, 2009.

[50] S. S. Bielack, B. Kempf-Bielack, G. Delling et al., "Prognostic factors in high-grade osteosarcoma of the extremities or trunk: an analysis of 1,702 patients treated on neoadjuvant cooperative osteosarcoma study group protocols," Journal of Clinical Oncology, vol. 20, no. 3, pp. 776-790, 2002.

[51] C. E. García Franco, W. Torre, A. Tamura et al., "Longterm results after resection for bone sarcoma pulmonary metastases," European Journal of Cardiothoracic Surgery, vol. 37, no. 5, pp. 1205-1208, 2010.

[52] G. Bacci, S. Ferrari, F. Bertoni et al., "Prognostic factors in nonmetastatic Ewing's sarcoma of bone treated with adjuvant chemotherapy: analysis of 359 patients at the Istituto Ortopedico Rizzoli," Journal of Clinical Oncology, vol. 18, no. 1, pp. 4-11, 2000.

[53] S. J. Cotterill, S. Ahrens, M. Paulussen et al., "Prognostic factors in Ewing's tumor of bone: analysis of 975 patients from the European Intergroup Cooperative Ewing's Sarcoma Study Group," Journal of Clinical Oncology, vol. 18, no. 17, pp. 3108-3114, 2000.

[54] G. Bacci, C. Forni, A. Longhi et al., "Long-term outcome for patients with non-metastatic Ewing's sarcoma treated with adjuvant and neoadjuvant chemotherapies. 402 patients treated at Rizzoli between 1972 and 1992," European Journal of Cancer, vol. 40, no. 1, pp. 73-83, 2004.

[55] M. Paulussen, S. Ahrens, A. W. Craft et al., "Ewing's tumors with primary lung metastases: survival analysis of 114 
(European intergroup) cooperative Ewing's sarcoma studies patients," Journal of Clinical Oncology, vol. 16, no. 9, pp. 3044-3052, 1998.

[56] A. Y. Giuffrida, J. E. Burgueno, L. G. Koniaris, J. C. Gutierrez, R. Duncan, and S. P. Scully, "Chondrosarcoma in the United States (1973 to 2003): an analysis of 2890 cases from the SEER database," Journal of Bone and Joint Surgery. Series A, vol. 91, no. 5, pp. 1063-1072, 2009.

[57] E. O. Burgert Jr., M. E. Nesbit, L. A. Garnsey et al., "Multimodal therapy for the management of nonpelvic, localized Ewing's sarcoma of bone: intergroup study IESSII," Journal of Clinical Oncology, vol. 8, no. 9, pp. 1514-1524, 1990.

[58] P. Picci, M. Mercuri, S. Ferrari et al., "Survival in highgrade osteosarcoma: improvement over 21 years at a single institution," Annals of Oncology, vol. 21, no. 6, pp. 1366-1373, 2009.

[59] M. P. Coleman, P. Babb, P. Damiecki et al., Cancer Survival Trends in England and Wales, 1971-1995 Deprivation and NHS Region, The Stationery Office, London, UK, 1999.

[60] C. A. Stiller, S. J. Passmore, M. E. Kroll, P. A. Brownbill, J. C. Wallis, and A. W. Craft, "Patterns of care and survival for patients aged under 40 years with bone sarcoma in Britain, 1980-1994," British Journal of Cancer, vol. 94, no. 1, pp. 2229, 2006.

[61] G. N. Machak, S. I. Tkachev, Y. N. Solovyev et al., "Neoadjuvant chemotherapy and local radiotherapy for high-grade osteosarcoma of the extremities," Mayo Clinic Proceedings, vol. 78, no. 2, pp. 147-155, 2003.

[62] S. Patel and T. F. DeLaney, "Advanced-technology radiation therapy for bone sarcomas," Cancer Control, vol. 15, no. 1, pp. 21-37, 2008.

[63] T. F. DeLaney, N. J. Liebsch, F. X. Pedlow et al., "Phase II study of high-dose photon/proton radiotherapy in the management of spine sarcomas," International Journal of Radiation Oncology Biology Physics, vol. 74, no. 3, pp. 732739, 2009.

[64] Y. Chen, F. Ciernik, A. Niemierko et al., "Treatment of unresectable osteosarcoma with proton therapy," International Journal of Radiation Oncology, vol. 75, no. 3, Abstract 2770, 2009.

[65] C. Blattmann, S. Oertel, D. Schulz-Ertner et al., "Nonrandomized therapy trial to determine the safety and efficacy of heavy ion radiotherapy in patients with non-resectable osteosarcoma," BMC Cancer, vol. 10, article no. 96, 2010.

[66] G. Noël, L. Feuvret, R. Ferrand, G. Boisserie, J.-J. Mazeron, and J.-L. Habrand, "Radiotherapeutic factors in the management of cervical-basal chordomas and chondrosarcomas," Neurosurgery, vol. 55, no. 6, pp. 1252-1260, 2004.

[67] G. Bacci, E. Palmerini, E. L. Staals et al., "Ewing's sarcoma family tumors of the humerus: outcome of patients treated with radiotherapy, surgery or surgery and adjuvant radiotherapy," Radiotherapy and Oncology, vol. 93, no. 2, pp. 383387, 2009.

[68] A. Schuck, S. Ahrens, M. Paulussen et al., "Local therapy in localized Ewing tumors: results of 1058 patients treated in the CESS 81, CESS 86, and EICESS 92 trials," International Journal of Radiation Oncology Biology Physics, vol. 55, no. 1, pp. 168-177, 2003.

[69] M. E. Nesbit Jr., E. A. Gehan, E. O. Burgert Jr. et al., "Multimodal therapy for the management of primary, nonmetastatic Ewing's Sarcoma of bone: a long-term follow-up of the first intergroup study," Journal of Clinical Oncology, vol. 8, no. 10, pp. 1664-1674, 1990.
[70] A. McTiernan, D. Driver, M. P. Michelagnoli, A. M. Kilby, and J. S. Whelan, "High dose chemotherapy with bone marrow or peripheral stem cell rescue is an effective treatment option for patients with relapsed or progressive Ewing's sarcoma family of tumours," Annals of Oncology, vol. 17, no. 8, pp. 13011305,2006

[71] J. A. M. Bramer, A. A. Abudu, R. J. Grimer, S. R. Carter, and R. M. Tillman, "Do pathological fractures influence survival and local recurrence rate in bony sarcomas?" European Journal of Cancer, vol. 43, no. 13, pp. 1944-1951, 2007.

[72] I. J. Lewis, M. A. Nooij, J. Whelan et al., "Improvement in histologic response but not survival in osteosarcoma patients treated with intensified chemotherapy: a randomized phase III trial of the European osteosarcoma intergroup," Journal of the National Cancer Institute, vol. 99, pp. 112-128, 2007.

[73] I. J. Cohen, "High-dose methotrexate is effective in osteosarcoma so what is the problem?" Journal of Pediatric Hematology/Oncology, vol. 31, no. 12, pp. 892-894, 2009.

[74] S. Ferrari, S. Smeland, M. Mercuri et al., "Neoadjuvant chemotherapy with high-dose ifosfamide, high-dose methotrexate, cisplatin, and doxorubicin for patients with localized osteosarcoma of the extremity: a joint study by the italian and Scandinavian Sarcoma Groups," Journal of Clinical Oncology, vol. 23, no. 34, pp. 8845-8852, 2005.

[75] S. Ferrari, S. Smeland, A. Bielack et al., Journal of Clinical Oncology, vol. 27, supplement, p. 15s, 2009, abstract 10516, 2009 ASCO Annual Meeting.

[76] E. C. Van Dalen and B. Canargo, "Mthotrexate for high-grade osteosarcoma in children and young adults," in The Cochrane Collaboration, John Wiley and Sons, New York, NY, USA, 2009.

[77] A. M. Goorin, D. J. Schwartzentruber, M. Devidas et al., "Presurgical chemotherapy compared with immediate surgery and adjuvant chemotherapy for nonmetastatic osteosarcoma: pediatric oncology group study POG-8651," Journal of Clinical Oncology, vol. 21, no. 8, pp. 1574-1580, 2003.

[78] P. Picci, L. Sangiorgi, B. T. Rougraff, J. R. Neff, R. Casadei, and M. Campanacci, "Relationship of chemotherapy-induced necrosis and surgical margins to local recurrence in osteosarcoma," Journal of Clinical Oncology, vol. 12, no. 12, pp. 2699_ 2705, 1994.

[79] P. A. Meyers, C. L. Schwartz, M. D. Krailo et al., "Osteosarcoma: the addition of muramyl tripeptide to chemotherapy improves overall survival-a report from the children's oncology group," Journal of Clinical Oncology, vol. 26, no. 4, pp. 633-638, 2008.

[80] L. Kager, A. Zoubek, U. Pötschger et al., "Primary metastatic osteosarcoma: presentation and outcome of patients treated on neoadjuvant Cooperative Osteosarcoma Study Group protocols," Journal of Clinical Oncology, vol. 21, no. 10, pp. 2011-2018, 2003.

[81] S. Ferrari, A. Briccoli, M. Mercuri et al., "Postrelapse survival in osteosarcoma of the extremities: prognostic factors for long-term survival," Journal of Clinical Oncology, vol. 21, no. 4, pp. 710-715, 2003.

[82] B. Kempf-Bielack, S. S. Bielack, H. Jürgens et al., "Osteosarcoma relapse after combined modality therapy: an analysis of unselected patients in the Cooperative Osteosarcoma Study Group (COSS)," Journal of Clinical Oncology, vol. 23, no. 3, pp. 559-568, 2005.

[83] B. Massimo, G. Giovanni, F. Stefano et al., "Phase 2 trial of two courses of cyclophosphamide and etoposide for relapsed 
high-risk osteosarcoma patients," Cancer, vol. 115, no. 13, pp. 2980-2987, 2009.

[84] A. Muñoz, J. Alfaro, N. Pardo et al., "Long-term results of the Spanish Protocol SO-95 for the treatment of nonmetastatic high-grade osteosarcoma of the extremities in children," Clinical and Translational Oncology, vol. 11, no. 6, pp. 387-392, 2009.

[85] M. L. Bernstein, M. Devidas, D. Lafreniere et al., "Intensive therapy with growth factor support for patients with Ewing tumor metastatic at diagnosis: pediatric oncology group/children's cancer group phase II study 9457-a report from the children's oncology group," Journal of Clinical Oncology, vol. 24, no. 1, pp. 152-159, 2006.

[86] H. E. Grier, M. D. Krailo, N. J. Tarbell et al., "Addition of ifosfamide and etoposide to standard chemotherapy for Ewing's sarcoma and primitive neuroectodermal tumor of bone," New England Journal of Medicine, vol. 348, no. 8, pp. 694-701, 2003.

[87] H. Jurgens, U. Exner, H. Gadner et al., "Multidisciplinary treatment of primary Ewing's sarcoma of bone: a 6-year experience of a European Cooperative trial," Cancer, vol. 61, no. 1, pp. 23-32, 1988.

[88] M. Paulussen, A. W. Craft, I. Lewis et al., "Results of the EICESS-92 study: two randomized trials of Ewing's sarcoma treatment—cyclophosphamide compared with ifosfamide in standard-risk patients and assessment of benefit of etoposide added to standard treatment in high-risk patients," Journal of Clinical Oncology, vol. 26, no. 27, pp. 4385-4393, 2008.

[89] C. R. Pinkerton, A. Bataillard, S. Guillo, O. Oberlin, B. Fervers, and T. Philip, "Treatment strategies for metastatic Ewing's sarcoma," European Journal of Cancer, vol. 37, no. 11, pp. 1338-1344, 2001.

[90] L. M. Wagner, N. McAllister, R. E. Goldsby et al., "Temozolomide and intravenous irinotecan for treatment of advanced Ewing sarcoma," Pediatric Blood and Cancer, vol. 48, no. 2, pp. 132-139, 2007.

[91] S. Burdach, A. Meyer-Bahlburg, H. J. Laws et al., "Highdose therapy for patients with primary multifocal and early relapsed Ewing's tumors: results of two consecutive regimens assessing the role of total-body irradiation," Journal of Clinical Oncology, vol. 21, no. 16, pp. 3072-3078, 2003.

[92] 2010, http://www.clinicaltrials.gov/.

[93] J. S. Whelan, R. J. Burcombe, J. Janinis, A. M. Baldelli, and A. M. Cassoni, "A systematic review of the role of pulmonary irradiation in the management of primary bone tumours," Annals of Oncology, vol. 13, no. 1, pp. 23-30, 2002.

[94] A. Cangir, T. J. Vietti, E. A. Gehan et al., "Ewing's sarcoma metastatic at diagnosis. Results and comparisons of two Intergroup Ewing's Sarcoma Studies," Cancer, vol. 66, no. 5, pp. 887-893, 1990.

[95] P. J. Leavey, L. Mascarenhas, N. Marina et al., "Prognostic factors for patients with Ewing sarcoma (EWS) at first recurrence following multi-modality therapy: a report from the children's oncology group," Pediatric Blood and Cancer, vol. 51, no. 3, pp. 334-338, 2008.

[96] A. M. McTiernan, A. M. Cassoni, D. Driver, M. P. Michelagnoli, A. M. Kilby, and J. S. Whelan, "Improving outcomes after relapse in Ewing's sarcoma: analysis of 114 patients from a single institution," Sarcoma, vol. 2006, Article ID 83548, 8 pages, 2006.

[97] M. Cesari, F. Bertoni, P. Bacchini, M. Mercuri, E. Palmerini, and S. Ferrari, "Mesenchymal chondrosarcoma. An analysis of patients treated at a single institution," Tumori, vol. 93, no. 5, pp. 423-427, 2007.
[98] T. M. Dantonello, C. Int-Veen, I. Leuschner et al., "Mesenchymal chondrosarcoma of soft tissues and bone in children, adolescents, and young adults: experiences of the CWS and COSS study groups," Cancer, vol. 112, no. 11, pp. 2424-2431, 2008.

[99] S. Boriani, S. Bandiera, R. Biagini et al., "Chordoma of the mobile spine: fifty years of experience," Spine, vol. 31, no. 4, pp. 493-503, 2006.

[100] D. Schulz-Ertner, A. Nikoghosyan, C. Thilmann et al., "Results of carbon ion radiotherapy in 152 patients," International Journal of Radiation Oncology Biology Physics, vol. 58, no. 2, pp. 631-640, 2004.

[101] T. F. DeLaney, N. J. Liebsch, F. X. Pedlow et al., "Phase II study of high-dose photon/proton radiotherapy in the management of spine sarcomas," International Journal of Radiation Oncology Biology Physics, vol. 74, no. 3, pp. 732739, 2009.

[102] S. Stacchiotti, A. Marrari, E. Tamborini et al., "Response to imatinib plus sirolimus in advanced chordoma," Annals of Oncology, vol. 20, no. 11, pp. 1886-1894, 2009.

[103] E. P. Buddingh, J. K. Anninga, M. I.M. Versteegh et al., "Prognostic factors in pulmonary metastasized high-grade osteosarcoma," Pediatric Blood and Cancer, vol. 54, no. 2, pp. 216-221, 2010.

[104] H. C. Holscher, J. L. Bloem, H.-J. Van der Woude et al., "Can MRI predict the histopathological response in patients with osteosarcoma after the first cycle of chemotherapy?" Clinical Radiology, vol. 50, no. 6, pp. 384-390, 1995.

[105] A. Abudu, A. M. Davies, P. B. Pynsent et al., "Tumour volume as a predictor of necrosis after chemotherapy in Ewing's sarcoma," Journal of Bone and Joint Surgery. Series B, vol. 81, no. 2, pp. 317-322, 1999.

[106] L. H. Aksnes, H. C.F. Bauer, A. A. Dahl et al., "Health status at long-term follow-up in patients treated for extremity localized Ewing sarcoma or osteosarcoma: a Scandinavian Sarcoma Group Study," Pediatric Blood and Cancer, vol. 53, no. 1, pp. 84-89, 2009.

[107] T. Langer, W. Stöhr, M. Paulides et al., "Prospective multicenter registration of major late sequelae in sarcoma patients using the Late Effects Surveillance System (LESS)," Klinische Padiatrie, vol. 217, no. 3, pp. 176-181, 2005.

[108] M. Bassal, A. C. Mertens, L. Taylor et al., "Risk of selected subsequent carcinomas in survivors of childhood cancer: a report from the childhood cancer survivor study," Journal of Clinical Oncology, vol. 24, no. 3, pp. 476-483, 2006.

[109] C. Rodriguez-Galindo, C. Poquette, N. Marina et al., "Hematologic abnormalities and acute myeloid leukemia in children and adolescents administered intensified chemotherapy for the Ewing sarcoma family of tumours," Journal of Pediatric Hematology/Oncology, vol. 22, no. 4, pp. 321-329, 2000. 


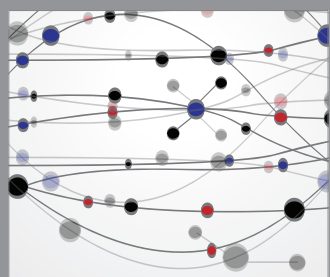

The Scientific World Journal
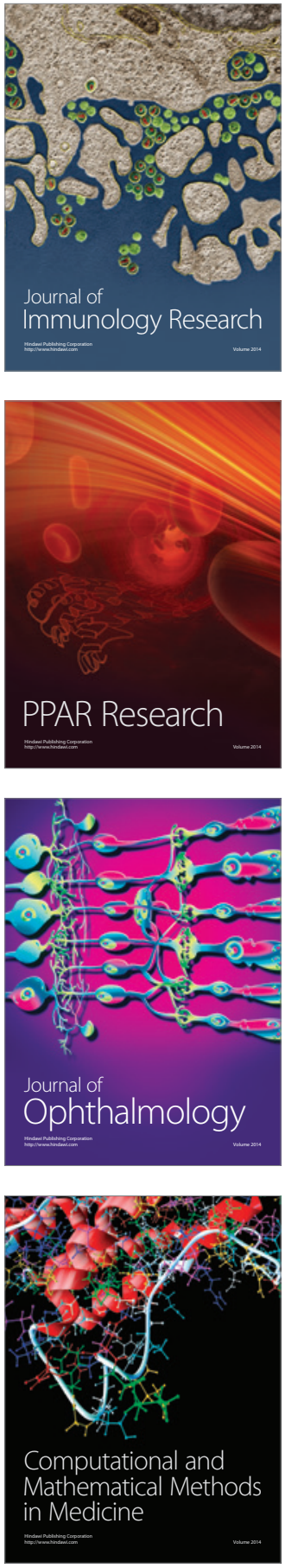

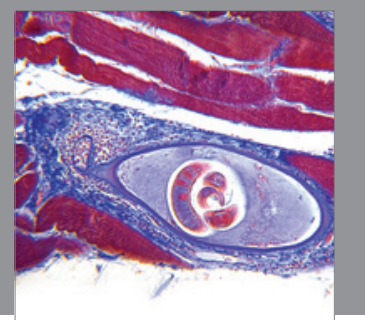

Gastroenterology

Research and Practice
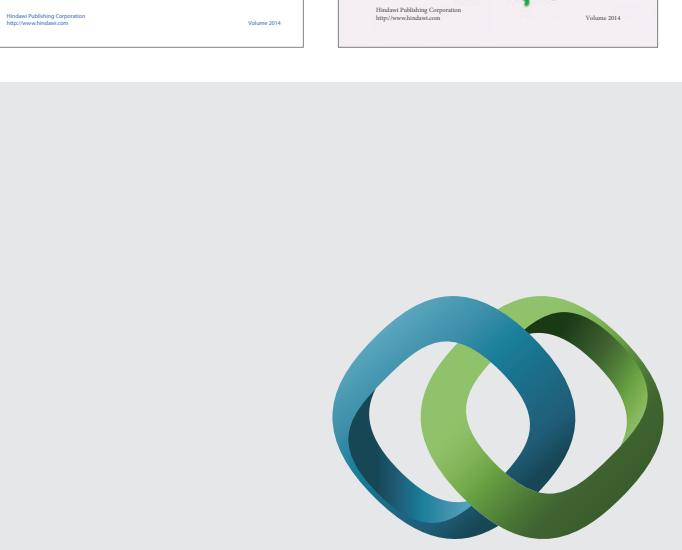

\section{Hindawi}

Submit your manuscripts at

http://www.hindawi.com
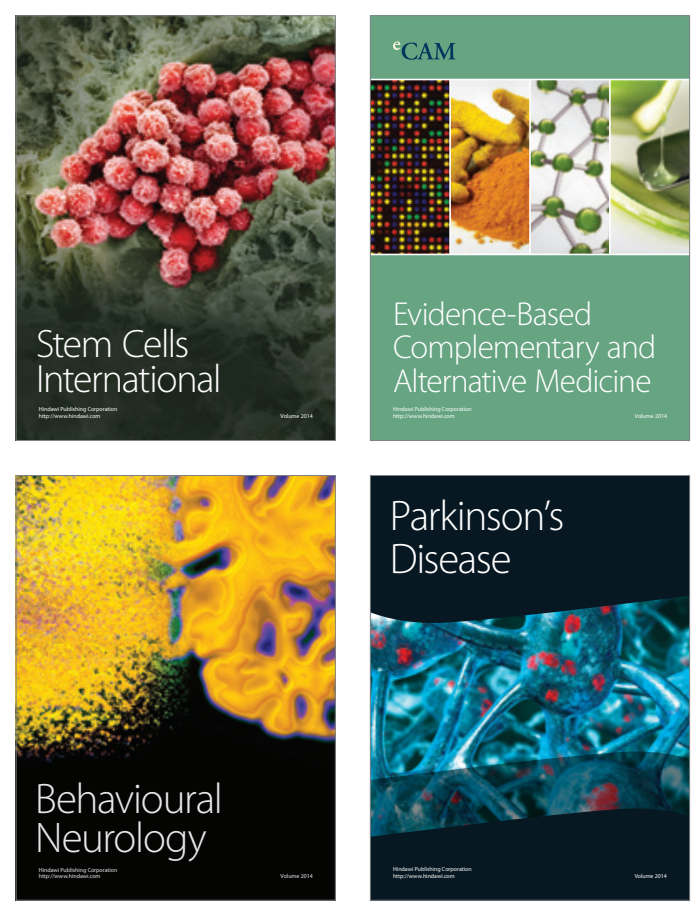

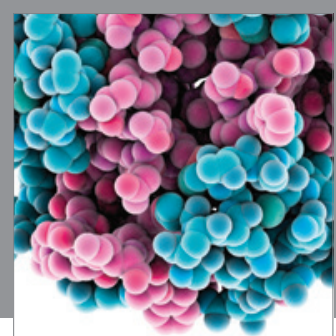

Journal of
Diabetes Research

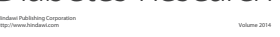

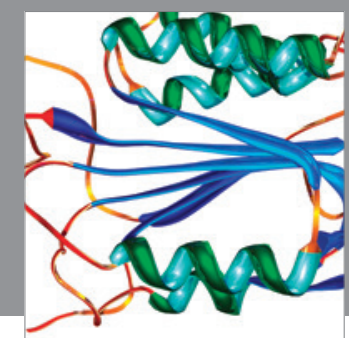

Disease Markers
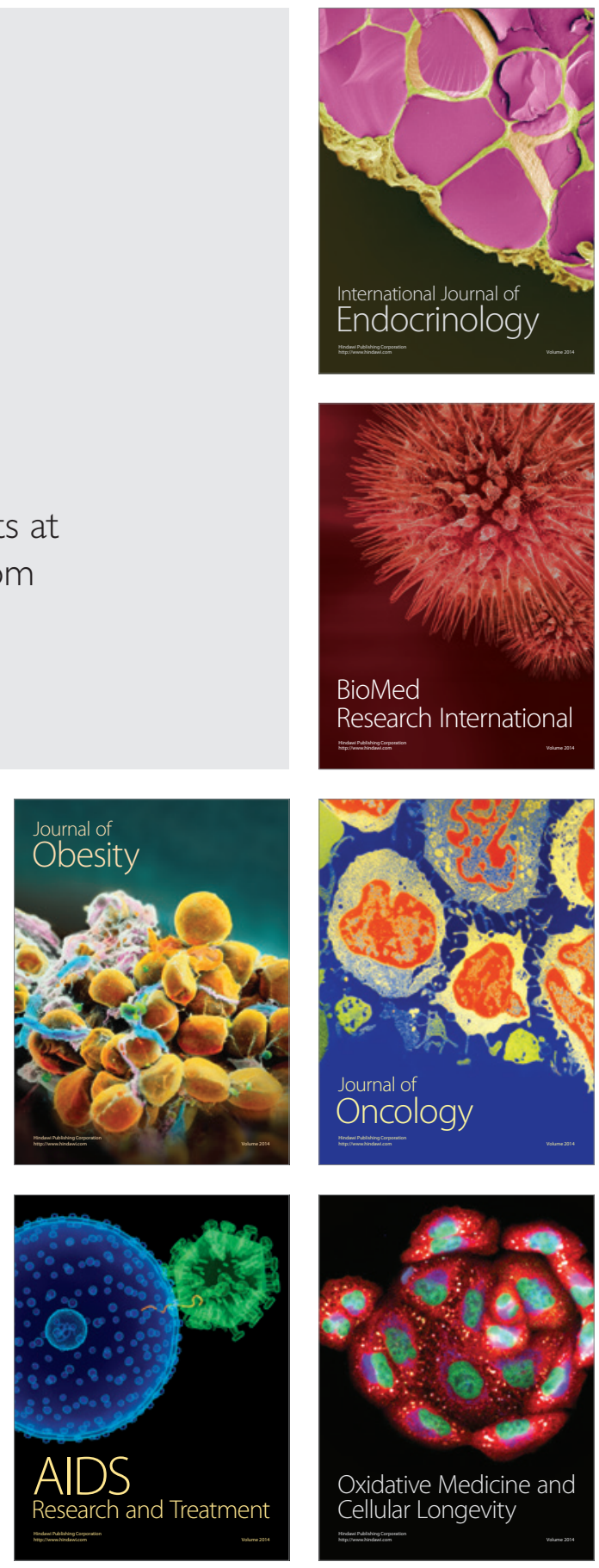